\title{
THE MODERN THEORY OF THE CELL AS A COMPLEX OF ORGANIZED UNITS
}

\author{
By E. W. Roberts.
}

\section{CONTENTS}

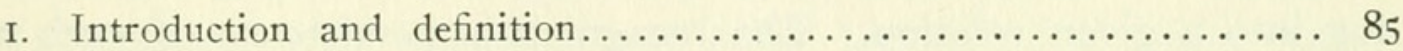

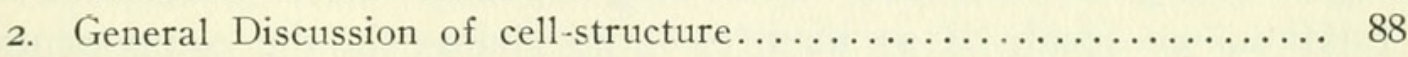

3. Illustration of such unities of decreasing value.............. 89

4. A comparative study of cells ............................ 89

Muscle fibrils, as differentiated within the cell.............. 90

Theory of the ebb and flow of units between nucleus and reticulum 92

Mutual stimulation of cells in tissues and its results.......... 93

Qualitative differentiation of cells, leading to mutants and tissues.. 94

Effects of varying food upon cells and cell parts............. 94

Yolk formation in eggs and embryonic tissue............... 94

Degeneration of larval tissue into foods................. 96

Some Special Cell Structures: Pigment Masses, Pseudopodia, Etc. 96

Epidermal cells and their products.................... 97

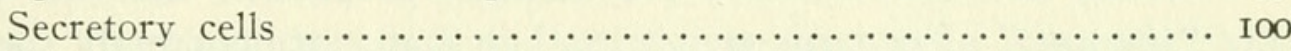

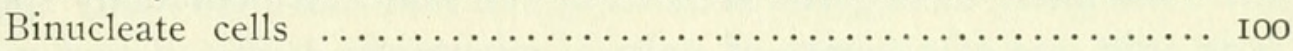

Formation of walls and skeletons by cells................. Ior

Nerve cells $\ldots \ldots \ldots \ldots \ldots \ldots \ldots \ldots \ldots \ldots \ldots \ldots \ldots \ldots \ldots \ldots \ldots \ldots$ Iо

5. The theory of the interrelation of nuclear and cytoplasmic units..... I02

Illustrated by Rhizopods ........................... I03

Illustrated by Spirogyra.......................... I05

\section{INTRODUCTION AND DEFINITION}

The matter herein is not presented in a dogmatic, but rather in a suggestive, spirit. It is believed that the photographs present evidence in support of the views advanced.

*The writer wishes to extend his thanks to all who have aided in the preparation of this work. In an especial way he wishes to express his obligation to Dr. S. G. Shanks of Albany, N. Y., and Mr. Frank Dienelt, Loda, Ill., both deceased. Dr. Shanks was much interested in the theories advanced in the paper and prepared some 800 slides of serial sections from which these cytological photographs were made. Mr. Dienelt introduced the writer to beauties of insect microscopy. This great hearted farmer, microscopist, and astronomer was, for more than 60 years, an arduous worker in the advancement of his favorite studies. During this time he made upward of 20,000 microscopic slides illustrating insect structure. 
The writer has found the view that the cell is composed of smaller units, each of which has a somewhat sharply defined work, of great value in forming a comprehensive mental picture of living matter. While this view, as opposed to that which regards protoplasm as a definite substance, is in no sense new and is the one held by most cytologists, the writer believes that the real significance of this newer view has not been fully realized by students.

Life is not to be looked upon as a force or a principle, but rather as the sum total of the component materials and forces entering into a given relation. The recognizable persistence of such a relation, we speak of as its life. Any change in the components entering into a given relation produces a corresponding change in the net result. This accounts for the constant change in the expression of all organic and inorganic groups, which is called evolution.

Text-books have taught for the last fifty years that the cell is "the unit" of such "living," organic structure; and that it is composed of a mystic jelly substance called protoplasm, which jelly (by powers equally mystic) is capable of carrying on all the varied processes of life.

Many modern workers are inclined to believe that the cell is not "the unit" of organic structure, but that more correctly speaking it is only one of a series of natural groupings, being in fact a whole household by itself, made up of smaller and smaller groupings of units governed entirely by natural chemical and physical conditions.

In the spirit of this conception the cell, which is in truth a convenient unit, may be described as a series of groupings of structures, increasing in complexity upward, arranged by past environment, and capable of modification by present environment.

Certain of these smaller units that make up the cell are clearly recognizable with the technic of the day; some of them at the other extreme are of the order of the molecule, with which the chemist must acquaint us; doubtless there are still other intermediate units lying between these extremes about which, at the present moment, we can only speculate, since they are too minute for microtechnic and too complex and multiform for satisfactory chemical analysis.

For more satisfactory approach to the problems involved I propose the following analysis of the cell structure; and taking the cell 
as the "unit" of study we may conceive the following order of subordinate units :

I. Intra-cellular units of the first order:-the nucleus; the cytoplasmic mass; and possibly the centrosphere. These seem to be somewhat coordinate in rank and subject to recognizable interactions.

2. Intra-cellular units of the second order:-(a) Within nucleus-chromosomes, spindle fibrils, etc.; (b) without nucleus (cytoplasm) - chloroplastids and other plastids, pyrenoids, centrospheres, etc. As a collective, descriptive term for the various kinds of units of this order, within the nucleus, I propose the term nucleosome; for those constituting the cytoplasm, the term cytosome. In the light of our ignorance of just what portions of the protoplasm do the particular things done within it, it is desirable to have such a term referring to the complex and composite nature of protoplasm in order to prevent us looking upon these various products as formed by the whole body of matter as a unitary mass. It is the thesis of this paper that work of the cell is done by the differentiated units of which the cell is composed, rather than by a "substance."

3. Intra-cellular units of the third order. Our analysis of this order of unit halts and awaits a more perfect technic. We have, however, gone far enough to feel that the chromosomes of the nucleus are composed of bodies-granules-arranged with some definiteness upon the linin thread-work. Whether there is a similar differentiation in other cell units we cannot say certainly from observation.

4. Intra-cellular units of the $\mathrm{n}^{\text {th }}$. order. Here belong the chemical unities of the order of the molecule. It is not the purpose of this paper to discuss the units of this class. We can only say that the characteristics of the higher units come from the character and relations of these; and that there are probably several series of ascending units between these and those suggested under 3 above.

Some of these subordinate units at least are endowed with one particular distinguishing quality: they undergo binary division at certain periods of their growth. It seems probable indeed that this is the process by which all organic groups multiply; and it is possible 
that this extends even throughout the ultimates of inorganic phenomena.

\section{GENERAL DISCUSSION OF CELL STRUCTURE}

The cell, as a rule, consists of a body composed of smaller units, which carry on all the vegetative processes of the group. This is usually termed the cyto-reticulum. This cyto-reticulum body contains an important structure called the nucleus, which plays an important part in the divisions of the cell, both direct and indirect. The nucleus, as we find it functioning in indirect divisions of the cells in higher forms of organic life, was not always present in the complexity in which it now exists. This is suggested by the fact that it is structurally much simpler in the lower types of cells.

It must have developed from its phylogenetic ancestors, just as is true of all higher organic structures. Its functioning as a compound division-organ may have arisen from the compounding of the binary division functions of lower organic units whose union gave rise to it.

The cell as we know it today, represents a line of descent from a remote geologic past, its present structure of nucleus and cytoplasm having grown from primitive groupings of lower and lower elements.

Furthermore the organic is but the inorganic in certain natural groupings. Life both comes into view and disintegrates from its groupings by natural processes. The more we know both of organic and inorganic, the more reasonable becomes the assumption that the principle of the inorganic is the same as of the organic.

Strasburger states the matter in this way: "The original protoplasm had no nucleus, all its parts being capable of both formative and nutritive functions. Then there was a gradual separation of both formative and nutritive parts, and the formative parts were the first differentiated carriers of hereditary qualities.

At first they were scattered in the cytoplasm, but later they became grouped, as in the Cyanophyceae; next the nucleus would be marked off by a membrane. Simple constriction might suffice for the division of such a nucleus, but as the difference between the hereditary units became so great that each unit carried only one quality, a more exact division would become necessary. The units 
would become arranged longitudinally in a thread, where they would undergo doubling, and the longitudinal division of the thread would separate the products of that doubling."

\section{ILLUSTRATIONS OF SUCH UNITIES OF DECREASING VALUE}

All organic structures show arrangement comparable to this; these relations are determined by natural conditions and are seemingly but a recapitulation in all the groups.

A typical series might be thus enumerated: nation, state, county, city, person, cells, cytosomes, daughter cytosomes, etc., clear down to the negative electrical corpuscle which is now deemed by many to be close to the border-land between matter and energy.

In a similar way, a series of animal structures such as muscle serves well as an illustration. The muscles cover the skeleton, and give form to the body; the muscle group is made up of individual muscles, which in turn are made up of bundles of muscle cells; these muscle cells are constructed of intracellular fibrils, which in turn are formed of smaller units.

Such groupings might be further illustrated, but enough is shown, to illustrate the fact, that higher groups are all composed of smaller groups, extending indefinitely lower apparently than optical instruments alone will ever carry us.

\section{COMPARATIVE STUDY OF CELLS}

There is a crying need for comprehensive knowledge of the life history of the various types of cells. In order to be effective this must be comparative and be based on the three great phases of cell life, the complete ontogenetic stages through which a cell passes on its way to its natural end:-viz., its

$$
\begin{aligned}
& \text { Pro,-or growth,-stages. } \\
& \text { Meta,-or functioning,--stages. } \\
& \text { Ana,-or disintegration,_-stages. }
\end{aligned}
$$

The probable phylogenetic significance of the ontogenetic stages of derivation and development would form an interesting phase of the treatment,- numbers of types being brought together for comparison.

A knowledge of the chemistry and physics of the various types at different stages is badly needed, also of the relations of cell types 
to their neighboring cell types, and to their other environmental conditions; and as badly needed as any phase, is an understanding of the relation between the vegetative cytosome system and the nucleus.

This is a gold mine of the future; no better field lies before the young student of biology.

\section{Muscle Fibrils, as Differentiated Within the Cell}

That the cell can be extremely complicated, is well shown in figure 3 , of the long section of the multinucleated muscle cells of a spider's leg; it will be plainly seen that the cell cavity contains many nuclei, scores of them in some cases. These giant muscle cells may be degenerate muscle bundles in which the walls of the component cells are not developed. In such a case the structure is termed a syncytium.

The walls of these cells, of which a better view is had in a cross section (Fig. 4), are seen to be lined with long bundles of cytosomes, which arise in plastids derived from the nuclear reticulum.

The plastids from which such cellular fibrils originate are well shown in figure $I$, and a later stage of the same in figure 2, from the back thoracic muscles of the Tussock Moth. The female of the Tussock Moth is wingless, and as a result we may expect to find early atrophy of the back thoracic muscles which move the wings in this sex.

Figures I and 2 both represent the female of this moth at twoday intervals. In figure 2 all but two of the muscle cells show distinct signs of degeneration, being much shrunken.
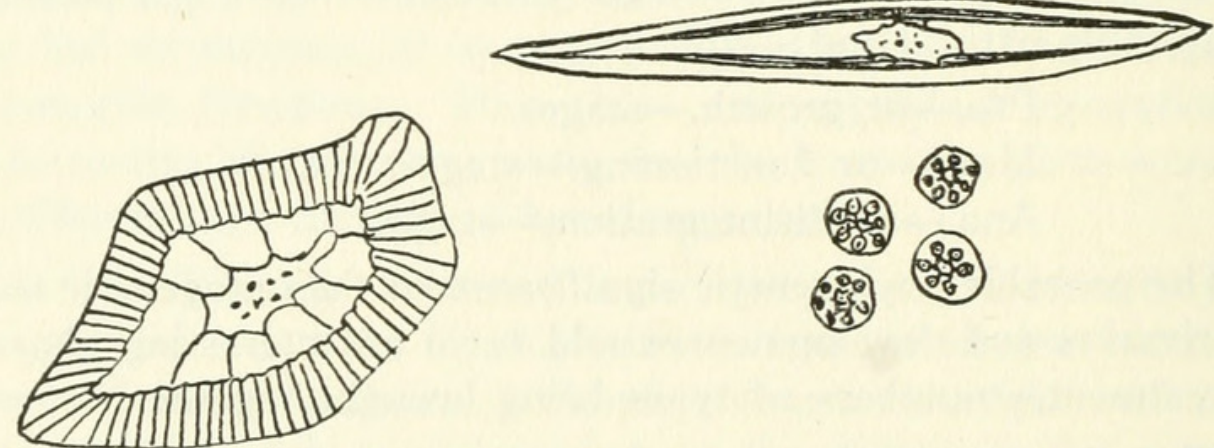

Fig. A.-The muscle cells in the leg of Spiders. The large cross section shows the fibrils arranged in flat radial plates: from the leg of Anglena.

The longitudinal sections and the small cross sections are in the early blast-stages, from embryo of Lycosa. 


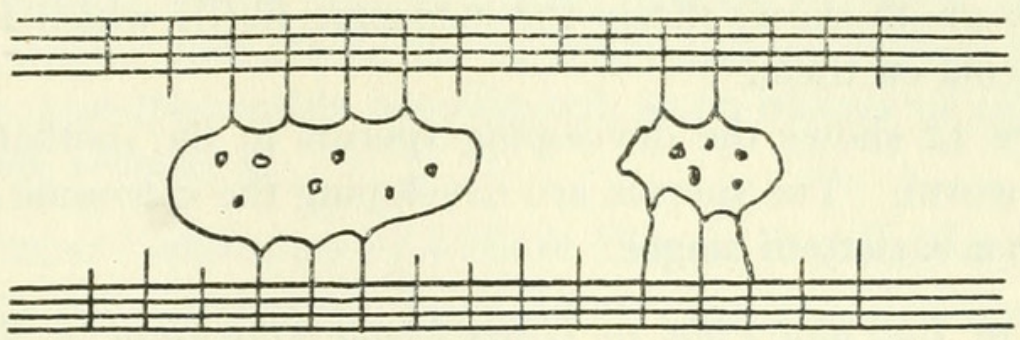

Fig. B.-Diagram of the nuclear attachments with the cytosome fibril system, in the leg muscles of a Spider, Agalena. Nuclear extensions are connected with the cell membranes.

Figure 5 shows the muscle-cells of a newly hatched frog tadpole's tail; the cytosome fibrils are in active stages of binary division. Many of the cells are multinucleated, and all these early stages of cells contain cytosome plastids of elaborated foods, commonly called the yolk grains; these are used as food for the growing fibrils.

Figure 6 is a long section of the back thoracic muscles of a fly pupa. Note the cyto-yolk grains and tracheal tubes between the muscle bundles; also the perimysium cells in the fibril bundles.

Figures 7 and 8 show long and cross sections of the developing cytosome fibrils in a Salamander pup. Note the increasing bulk by binary divisions of the fibrils.

It has been taught for years that the contractile and extensile powers of cells are due to what was termed "the general contractility of protoplasm"; this is evidently an error, as close observation will show that the motile and contractile powers are due to specific cytosome structures, in many cases in the form of fibrils.

The tails of sperms, all muscles, cilia, flagella, dendrites, psendopodia, nerve fibrils, etc., are moved by contractile cytosomes.

In figures 9-Io the vegetative stages of sperm development are shown. They are from the testicle of a salamander. The entire shape of the cell and of its appendages is here determined; the motile tail has cytosomes which both form and move it.

All the Protozoa or single cell animals and plants are extremely complicated, and have the cytosome structures elaborately diversified in function, as contractile and sensory fibrils, secretive and excretive organs, and in some cases sexual organs; they are not the simple jelly masses often described in text books.

Figure II is a section of the intestinal cells of the Carpenter 
Ant. The photo shows nicely the cytosome fibrils which form and move the cilia on them.

Figure 12 shows the developing sperms in the mother-cells of the earth worm. The sperms are developing the cytosome fibrils in the tails in a variety of stages.

Theory of Ebb and Flow of Units From Reticulum to Nucleus

The cyto-reticulum cell-structures are extremely complex in nature, and they show modifications for the performance of a great variety of function in a single cell; the division of labor among them is in some cases as varied as a more highly organized metazoan group, each individual cell having need for more or less of all the physîcal requirements of the higher groups.

Paramecium is a good subject in which to study the diversity of cytosome structures in a single cell. It is to the variations in the arrangements and functions of these cytosomes, and their smaller component groups, that we owe the great variety of expression found in organic life.

They are the true vegetative body of the cell, and it is possible that their arrangement and character is transmitted to the nucleus by minute representatives of the vegetative groups.

These representatives are assembled on the nuclear reticulum during early pro-stages of indirect or mitotic division; during direct division of the cell these representatives are not used, as the entire vegetative body is directly divided.

As in a metazoan group we find the body characters are transmitted by the sex sells, it follows that these characters must have been received from some source before they could be reproduced or given out again. Thus we see a constant working of a process in both directions:- the nucleus constantly buds out the characters of the cell body, while the body characters as constantly modify the nuclear characters.

This principle may be illustrated from a yet higher grouping. A nation, state, city, etc., will be constantly modified by emigrants from other countries, states and cities, the modification depending on the relative proportions and strengths of the new factors, the whole assemblage being also as constantly modified by the total environment. 
In a similar way it may be argued that the cytosome body bears a corresponding relation to the nucleus; and we believe this point should be most thoroughly investigated, as no history of cell life can be complete without it.

\section{Mutual Stimulation of Cells in Tissues, and Its Results}

As, in the ontogenetic development of a metazoan group, new types and functions and groupings of cells constantly come into view, we suggest that such new types should arise in many cases, by some form of cell association,- - a semi-conjugational arrangement.

Phylogenetically we well know that many new types and varieties arise by conjugation. This is the advantage of sex and indirect division,--that we get greater variety, and as a result greater resource from uniting differing individuals, than from like individuals.

Such a mutually stimulating grouping of a non-conjugating nature is shown in figure $\mathrm{I} 3$, of the giant spines or sexual horns on a male fly; figure I4 is a photo of the giant nuclei of these horns, while figures 15 and 16 are vertical sections of giant spines from other parts of the fly's body.

We have examined the nuclei of hundreds of the giant spine cells on this pupa, and all alike show in section, various numbers of cells with their nuclei in contact.

The explanation that seems reasonable, is that many of these spines arose in their phylogenetic development as a result of mutations produced by mixing slightly varying individual cells of the same or allied varieties.

In figures 18 and 20 will be seen the so-called "nurse-cells" which aid in forming the ovum. These nurse-cells are evidently of the nature of the stimulating cells in these giant spine groups. Their function must be something more than supplying food, for they themselves have to be fed so they may attain their giant size. They are constant in number in a species; thus this variety of Ephemera has six, while the variety of Mosquito has four and a variety of Chironomous has only one.

The real food cells are evidently the jacket of small cells which surround the ovum. From these small cells come the chromidia which form the yolk cytosomes. 
There are also, in the embryo sac of all higher plants, various numbers of large cells cut out along with the ovum cell. This phenomenon is quite widespread, and it seems as if blast cells requiring large amounts of nutriment cannot encompass the task without other cells of large size in the group.

This is an interesting field for research.

There are various other conjugant cells in different tissues of these young animals that show similar arrangements; but this being a striking type, we offer it in hope of stimulating further investigation.

Qualitative Differentiation of Cells, Leading to Mutants and Tissues

The two cells resulting from mitotic division are not in all cases like their progenitor; they are in many cases progressive mutants. That is, the contents of the cells resulting from the divisions may differ much in their vegetative functions and in their subsequent development. This becomes very evident when we watch the development of their vegetative systems from the nuclosome divisions of the nucleolus. The nucleosomes show variations in number, shape, size, position, structure and functions.

There thus arises in metazoan tissues a constantly varying type of cells, which is supplemented in many cases, such as ferns and other lower plants, by well known semi-conjugational cell arrangements. This is usually known as apogamy.

These mutations have an ancestral origin.

Effects of Varying Food Upon Cells and Cell Parts

We also suggest that the new types of cytosomes which elaborate the yolk granules and other food products in these growing cells, may have a direct modifying action on the other cytosomes which use them for food.

Food and the quest for the same, has been a great factor in the development of organic life, and it is wise to investigate the ontogenetic development, to detect traces of such reaction.

\section{Yolk Formation in Eggs and Embryonic Tissues}

If the type of product to be elaborated in a cyto-plastid is of a 
food nature, the daughter cytosomes do not remain adherent as in fibril products, but lie freely in the plastid wall in a semifluid mass.

The yolk of all egg cells is formed primarily by these cytoplastid inbuddings; this is a very elaborate process, and spreads over the time of the growth of the egg, from the mitosis which cuts out the future sex cell, to the time of the maturity of the yolk products.

A fine type of the elaboration of yolk plastids is shown in figure I7, of the ova of a spider. Here the enormous growth of the yolk cyto-plastids is well depicted.

The sex nucleus of the egg takes little part in the yolk formation of insect eggs; there are special nutritive cells set apart, which furnish elaborated cytosome products. Some of these cytosome products continue their growth in the plasma of the sex cell, there forming anew from the pabulum the typical yolk-cells or plastids.

The nutritive cells form a typical ovilemma around the sex cell. A good type of this is shown in figure I8, of the young ova of an Ephemera, in which case six cells are situated around the micropyle, while a complete jacket of smaller cells form the egg membrane proper.

The yolk cyto-plastids present a close resemblance to true cell types; in some cases showing a cell wall, plasma, and a staining body resembling a nucleus. This body is capable of division and subdivision; see figure I9, of the same Ephemera ova further matured.

Another illustration of these yolk cyto-plastids is seen in figure 20 , of the nearly mature eggs of the mosquito.

The yolk of ova is thus seen to be a special product of certain food cells (the ovalemma), which live for the sole purpose of furnishing food for the future embryo.

In developing embryos there is constantly a part of the cells set aside to manufacture special foods for the growing tissue cells. This gives the growing embryo a constantly developing and changing diet, and this process will doubtless be found to have a phylogenetic derivation and significance. It gives a continually new and developing food environment, and must be a profound factor in the modification and growth of the ontogenetic series of types of cells and their groups. 


\section{Degeneration of Larval Tissue Into Foods}

After the larva is hatched from the egg, the yolk food supply will be found nearly exhausted, and an immediate new food supply must be had. This when obtained is constantly assimilated by the larval tissues, which re-elaborate therefrom according to their needs.

During this larval stage, there is an extensive storage of cytosome food products: fat, yolk ,etc., beside all the larval tissue which will become useless when the pupa stage is entered upon.

These larval tissues as fast as they become ontogenetically useless, suffer anatosis stages and thereby disintegrate into foods. There are countless variations of these processes. In many cases it is accomplished by phagocytosis, as in figure $2 \mathrm{I}$, from a moth pupa, and figure 22, from an ant pupa.

There are in many cases anatosis phases on a large scale, and in some cases reversion to primitive organic types will be found.

In a developing pupa such as the Carpenter Ant,-Camponotus, -we have a striking illustration of the great variety of types among the food cells. These types are all grown in certain hereditary locations, and have in each type certain elaborated food products, which mature at certain times, and have certain stages of growing cells to feed. When mature they either undergo anatosis where grown, or migrate by amoeboid movements, or in blood streams, to where they are to be usd.

In one type of food cell there are two large bilateral plastids developed, which dominate all the other plastids. The other plastids develop as a swarm of dark staining bodies on the reticulum of the nucleus, between the dominant plastids. (See Fig. 23.) In another type the plastids are developed in many equal sized bodies as shown in figure 24. In still another type of these food cells, they become detached and are carried in blood streams into the developing legs, etc., where they undergo anatosis reduction into blood pabulum and are used as food.

Some Special Cell Structures, Pigment Masses, Pseudopodia, Etc.

It should be kept in mind that cells with irregular outlines are hereditarily just as marked, and have just as natural groupings in their fundamental makeup, as the most symmetrical ones. A glance, 
for example, at the irregular outlines of the pigment cells of a salamander (Fig. 27), will show the apparent anastomosing of the pseudopodia; these reticulated appearances are caused by the branching of the groups of cytosomes, the branches becoming superimposed in the focal plane.

Figure 28 gives, for comparison, a view of a pigment cell from a young fish embryo, with the fibrils in a conventional radiating arrangement.

In a pigment cell the cyto-fibrils are self delineating; in an object as colorless as an Ameba in a living state, such structures are practically invisible, only proper fixing and staining reagents being able to show them. In fact, this is the reason so many writers have called them "structureless protoplasm."

In Polycistinæ, Foraminifera, Rhizopods, etc., the pseudopodia are sent out at certain hereditarily determined places in the cell wall, forming "arm holes" in the silica, lime and chitine cases. (See Fig. 29 and Fig. 30.) These arms are merely cytosome structures, which are capable of extension and contraction. Some writers figure the so-called spindles of these pseudopodia, but none seem to realize their value.

During life a continual flow of cell plasma with floating corpuscles of cytosome derivation, may be seen over and among the fibrils. This flowing, streaming and boiling of cytosome products is due apparently to chemical and physical changes which take place on the addition of various components, by which the chemical balances of the groups are all thrown out of place and new readjustments follow. This furnishes us with the processes which are spoken of collectively as life.

\section{Epidermal Cells and Their Products}

The markings on insect hairs and scales are all cytosome products, and they serve a variety of purposes, from mimicry to the gorgeous color effects seen in flies, bees, beetles, butterflies, etc. (See Fig. 3I, of the scales of Lepisma; and Fig. 32, of the cyto-fibrils in the walls of moth scales in cross section.) 


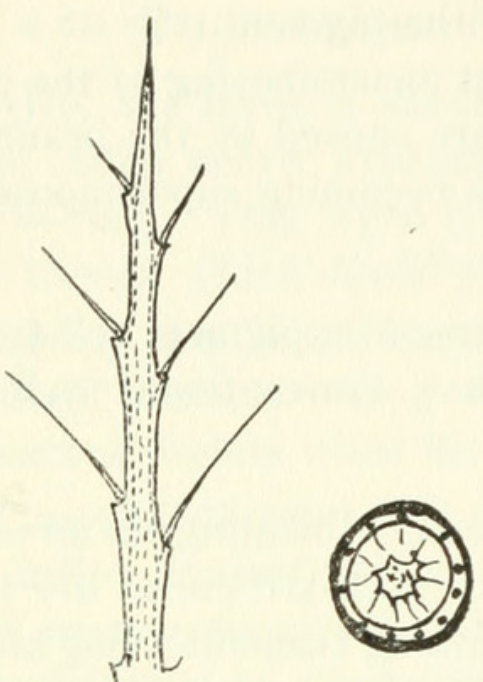

Fig. C.-Diagram of cross and longitudinal section of scale of Tussock moth, showing arrangement of fibrils.
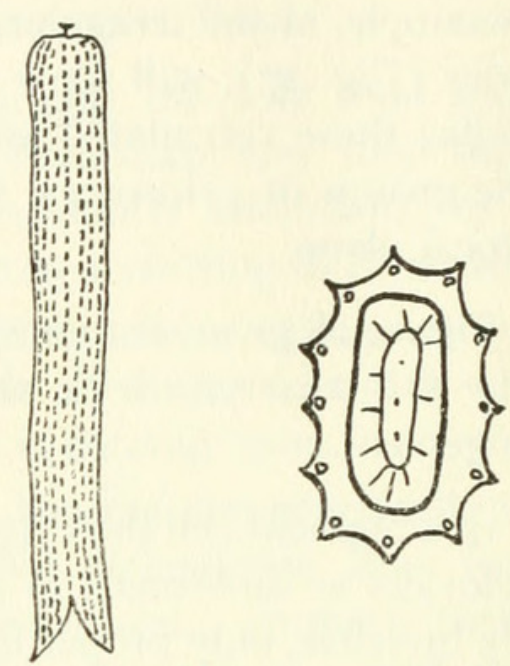

Fig. D.-Diagram of Branching spines of a spider, showing long and cross sections of the system of fibrils.

These scales are formed by vesicular sac-like extrusions of certain epidermal cells, and are caused by the growth and arrangement of the cytosome structures, which form groups of fibrils by binary divisions of the cytosomes remaining adherent in a longitudinal manner. These cyto-fibrils, by their aggregate extension, push the wall of the cell outward, forming a vesicle, in which the fibrils are plainly seen as the striations; later the vesicles are flattened or otherwise shaped, and the chitin of the cell wall becomes hardened.

In inscts which go through a pupal stage, very peculiar phenomena occur. When the larva is mature, and ready to enter the pupal state, the larval skin is ruptured and cast off. The insect now has the wings, legs, antennae, etc., well developed. They are folded by the insect ventrally, and the casting off of the larval skin ruptures the epiderm cells, which exude their cytosome products in a fluid state. This forms a coating of slime which rapidly oxidizes in the air, forming the pupal case.

The pupa now being protected by the pupa case, again elaborates its cytosome products in the epidermal cells, but this time they exude slowly, and form the scales as above. (See text Figs. C-G; and P1. 6. Figs. 3I-36.)

In figure 37 , we have a strongly stained view of the cytosomes 


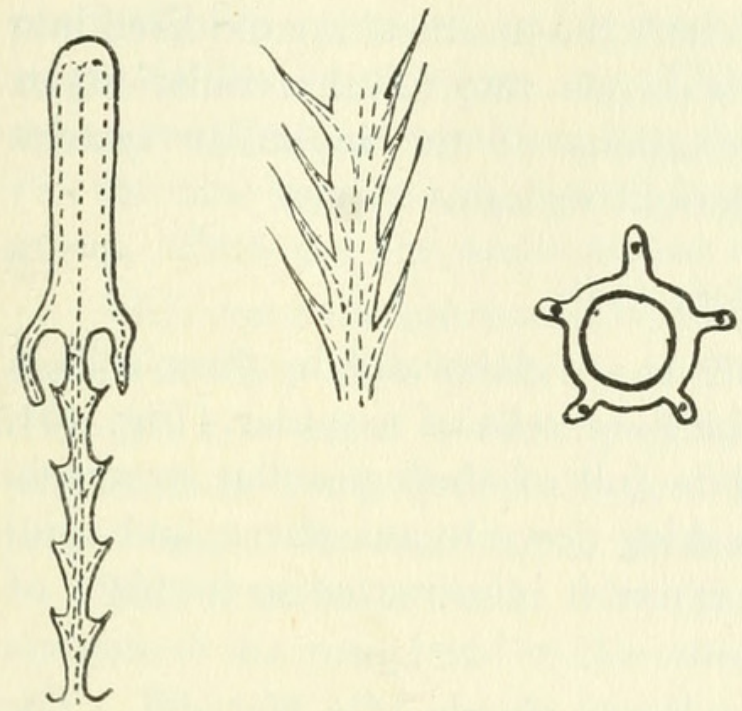

Fig. E.-Diagram of long and cross section of the cytosome fibrils in the spines of Trogdotyle larva. The spines have 5 heads or fibrils.

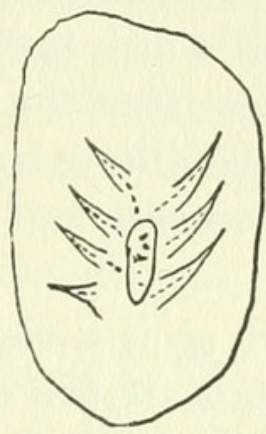

Fig. F.-Diagram of a multispined cell from the tongue of a Syrphus fly.

in the epidermal cells of a locust. By the extrusion of these cytosomes we get a variety of products: plain chitine, single scales, branching scales and multispined scales.

All the chitin structures of insects are formed by the oxidization of these various cytosome products. The diverse nature and groupings of these vegetative structures produce a multitude of external markings in organic life.

All the cell processes of assimilation and excretion are carried on by these cytosomes, and all the various animal and vegetable structures are the results of their groupings.

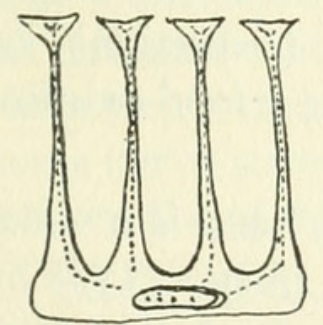

Fig. G.-Multi-spined cell from the pulvilli of a fly's foot; showing cytosome fibrils.

Figure 38 is a section of the spiral tracheal tubes of a butterfly larva, Mamestra picta. The tubes are of dermal origin and consist of a layer of hypodermal cells which pour out their cytosome prod- 
ucts into the lumen of the tubes, where the products are oxidized into a chitinous intima. This intima is thrown into spiral crenulations or threads by contraction and relaxations of the muscular system. These tubes sometimes contain dermal scales or spines.

\section{Secretory Cells}

In animals all the gland products are elaborated by these cells, a fine type of which is shown in the liver cells of a spider (Fig. 39). Here will be seen the cyto-plastids full of their peculiar products, while those on the margin are breaking down in ana-phase, and voiding their contents into the duct, where it is conveyed to its place of disposal.

Figure 40 is a view of the salivary glands of a Katydid. The large plastids are full of the peculiar semi-fluid cell-products.

\section{Binucleate Cells}

In some insects such as Apis, and some Hemiptera (See Fig. 24 ), the intestinal and fat cells will in many cases be found binucleated. It is believed that these nuclei are physiologically different. One nucleus, and its cytosomes, is vegetative undergoing anatosis, the elaborated products being discharged into the food mass in the intestine. This nucleus is as continually replaced by division of the other, which is a generative nucleus. This adaptation may be due to the quantities of fat used in wax and other products.

In most animals, however, the secreting cytosomes elaborate their products several times in succession; and finally the entire cell contents are voided, the cell being replaced by a new one.

During anatosis the cell products are disposed of in two ways; (I) voided into ducts and carried from the body (excretion), or (2) emptied into the blood circulation, there acting as a food or stimulus for other cells in a pro- or meta-stages.

Anatosis is sometimes called "self phagocytosis," and is a widespread function in the animal and vegetable world; phagocytosis by amoeboid cells may be considered as another phase of this phenomena.

Anatosis is the complement, or the opposite, of digestion; by the discharge of anatosis products into food masses the food is 
partically disorganized, and is then absorbed by other cells in a metastage, which re-elaborate it. They then undergo anatosis themselves, voiding the products into the lymphatic or the blood system (as the case may be), by which it is carried to the other cells of the group, which use the nourishment for pro- and meta-stages.

This constant succession of pro-, meta- and ana-phases of cytosome life keep alive the body of the person or cell group. Certain series of cytosomes constantly undergo ana-phases, that new groups may undergo pro and meta-stages. When the pro and meta-stages no longer equal the ana-stages, deterioration and death ensues.

This occurs naturally when the series of acquired hereditary* characters are completed in the ontogenetic development.

\section{Formation of Walls and Skeletons by Cells}

The cytosome membranes of many types of cells, which in many cases form the structural basis of cell walls, are often filled in with deposits of various substances, such as lime, silica, chitine, cellulose, etc. These substances do not easily decay, so we have many fine records left us of the arrangement of the original cytosomes which formed the membrane, in and on which the deposit was laid. Such elaborate deposits are well shown in Diatoms, Polycistinæ, Foramimfera, Sponge spicules, etc.

These cell walls all owe their peculiar form and structures to the minute (cytosome) groupings of the vegetative bodies of the cells.

\section{Nerve Cells}

Figure 43 is a section of the optic lobe of the brain of a moth. The multitude of curious extensions which nerve cells put forth are all actuated and formed by the cytosome groups, their various arrangements staining nicely; so intricate is the complex of these cytosome nerve structures that it is now deemed possible that there is not a fixed living cell in a metazoan body that is not reached by their

*Author's Note-It is believed that most students of the theory of Evolution will agree that organisms do acquire new characters, both in an ontogenetic and phylogenetic sense.

The various schools of Evolution merely differ as to the factors and their relative importance in producing the new aspects which organisms acquire.

We have carefully avoided taking sides in these arguments in this work, as they have an abundant literature of their own. 
extensions. It is due to the growth and multiplication of these extensions from the cytosome groups, that we get growth and increase of the cell group. How rapid this growth is will be seen when it is stated that in some insects the complete span of time from egg to mature insect is but a few days.

Figure 44 shows the growth of the cytosome fibrils in the brain cells of a Salamander.

Figures 45 and 46 show the active growing stages of the optical elements of a Tussock Moth eye. There are three days' difference between figures 45 and 46 ; the number of cells has not increased. The growth is entirely due to the enormous development of the cytosome systems.

Figure 42 shows a cross section of these cytosome fibril elements in the same eye.

\section{THEORY OF VEGETATIVE CELL STRUCTURES AND THEIR RELATION TO THE NUCLEUS}

The relation of the vegetative cyto-structures to the nucleus is of the utmost importance to a comprehensive understanding of the problem of organic life.

The structure of a typical cell in vegetative stage as conceived by the writer is given in the diagram below (text figure 8 ).

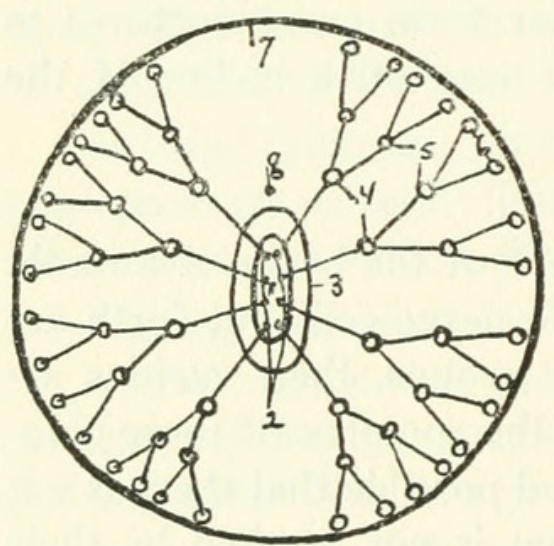

Fig. H.-Diagram designed to show the relation of nucleosomes and cytosomes in the cell. 1, nucleolus; 2, nuclear units-nucleosomes; 3, nuclear membrane; 4 , cytosomes or pyrenoids; 5 , daughter cytosomes; 6, grand-daughter cytosomes; 7 , cell wall; 8 , centrosome.

The cell and all its lower cytosome groupings are constantly arranged in halves of duplicate polarity. All these groups have definite family-numbers of components, a minimum point of greatest stability, and a maximum point of instability.

This is in accord with the discovery by Mendeleef of the peri- 
odic law of the elements; it has been possible for him to formulate the principal in accordance with which it is possible to group the various elements in the physico-chemical world into something like a harmonious scale.

The nucleus of a cell contains a body called the nucleolus; this body contains in various semi-fluid forms the balancing polarity groups of units. That is, if the vegetative group contains say fourteen negative pyrenoids, the nucleolus will contain a balancing group of fourteen positive units of equal value. If fourteen is the number at which the components of the group are at their greatest point of stability, an octave higher or double the number, or twenty-eight, will be the point of instability.

The growth of the cell from its point of stability to its adult point of instability is caused by a recapitulation of this same process in all the lower component groups; this continues until by binary divisions of all the cytosome groups, the stability of the larger cell groups is at its weakest point.

The nucleolus units are now attracted strongly by the negative vegetative groups, and this divides the nucleolus units into two groups of fourteen each. These immediately go toward their negative pyrenoid groups, and take their places in the middle of an equal number of vegetative units. Acomplete division of the cell contents is thus effected, both of the resultant cells being at the point of greatest stability.

\section{Illustrated by Rhizopods}

We cannot accept the view of Haeckel and other early writers that Rhizopods are the lowest of the forms of single cell life.

The Rhizopods are all parasitic or degenerate animal cells, which live on other organic matter, having lost the power of making their own food through their own chloroplast system.

Therefore we may expect to find the chloroplast system in all animal cells in a more or less rudimentary or specialized condition.

In all the Rhizopods the nucleus forms the chloroplast system by outbudding minute portions of nucleus matter called chromidia; these form all the vegetative parts of the cell.

By the fusion of several of these chromidia the new nuclei of a 
progeny of embryos or gametes are formed, the entire body being thus broken down into young.
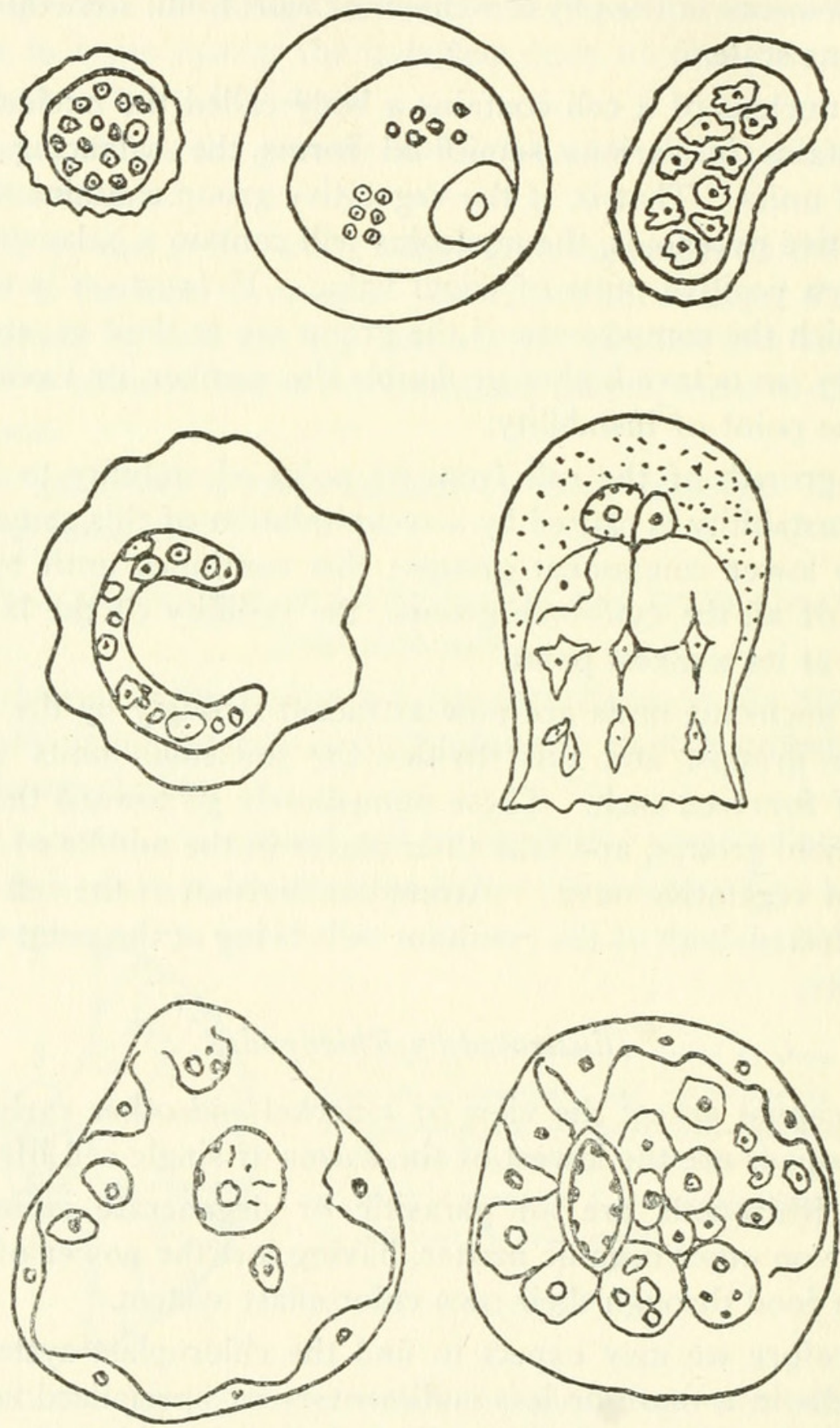

Fig. I.-Reproduction in Rhizopods.

See the works on Rhizopods by Lister, Calkins, Hertwig, Elpetiewsky and Siger. 
Now let us see if this production of plastids in all animal cells is not from similar chromidia fragments budded from the nucleus. In the cyto-plastids from an ant, Camponotus (Fig. 47), we see the swarm of chromidia emerging from the nucleus in all stages of growth. The larger plastids have various numbers of these chromidial granules, which take the nuclear stain very precisely.

Compare this cell with the figure of a Rhizopod by Hertwig (Fig. 44), and the resemblance is striking. There is this difference, however, in the Rhizopod: the plastids become detached as young, while in the food cell of the Camponotus they are specialized as food for growing tissues.

These chromidia are the nuclear elements of all the cytosome systems, and that they are entitled to be called cells in some cases is well shown by their ability to reform into definite nuclear structures.

Heidenhain's great work on "The Cell and Plasma" states that these chromidia have been found in all cell structures but muscle fibrils; here it is evidently diffused among other elements.

This agrees essentially with the numerous observations of the author. All the lower protozoa, both plants and animals, form their various swarm spores in this manner from chromidia.

In many cases the nucleus remains intact until after the spores are reieased, thus showing that the spores are not formed by its fragmentation.

\section{Illustrated by Spirogyra}

Spirogyra jugalis has a chromosome number of I4-28.

Several varieties of Spirogyra were examined, and the chromosome number of the type can be compared with the pyrenoid number of the different specimens examined.

Below are tabulated the counts of the pyrenoid numbers in many cells of each kind.

The pyrenoids should be counted only at adult stages or immediately after division; since between these points the pyrenoids are undergoing division and are indeterminate in number:

S. maxima, adult 6 bands, I4 pyrenoids in a band.

$S$. hassallii, adult 2 bands, 14 pyrenoids in a band.

S. porticalis, adult I band, 28 pyrenoids in a band. 
S. jugalis, adult 4 bands, 28 pyrenoids in a band.

S. fluviatilis, adult 4 bands, I4 pyrenoids in a band.

$S$. barians, I band, I4 pyrenoids in a band, adult.

S. tenuissima, I band, 56 pyrenoids in a band, adult.

$S$. condensata, I band, 7 pyrenoids in a band, adult.

S. setiformis, 4 bands, 7 pyrenoids in a band, adult.

The identification of the species may not be positive in all these cases ; the individual variation is so great it is almost impossible.

The number of chloroplast bands does not seem material, they simply represent divided pyrenoid groups; extra divisions, either way, among the pyrenoids does not affect the results.

In many plants the pyrenoid groups may be compounded, as in Zygnema and many Diatoms and Desmids.

(A diagram of No. 6 at adult and binary stage is given.)

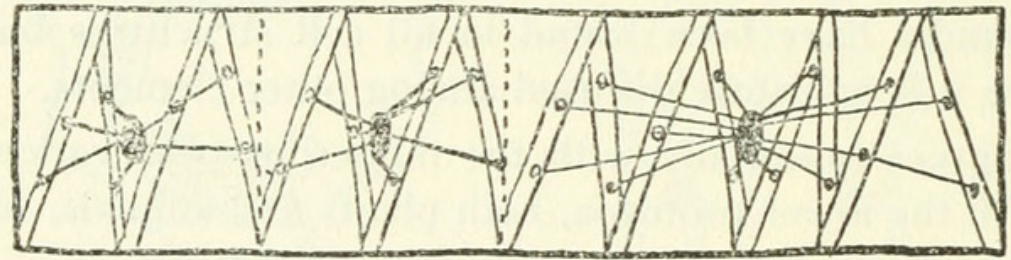

Fig. J.-Division in the cells of Spirogyra.

During life there is a connecting filament between each pyrenoid and the nucleus. Special effort is needed to determine whether these relations exist between the vegetative and nuclear groups of other types of cells as well. Here is a field almost untouched as yet.

For many years the vegetative division of the lower Algae was described as a binary division. Research with modern staining methods has developed the fact that the divisions are in reality but little different from the mitotic divisions of higher plants.

True binary division does occur, however, in many well certified cases; in such instances the nucleus seems to be the functionary organ.

During indirect or mitotic division, the vegetative body is represented on the nuclear reticulum, the nucleoli units migrate outward and are caught in the network of minor vegetative units.

These groups now undergo all their characteristic attractions 
and repulsions which form the pro-and meta-stages of mitosis, by which new cells get duplicate representation.

Cells which undergo binary division for long series of divisions and only at rare intervals resort to mitosis or conjugational division, use the nucleolus as a means of binary division; while the higher Metazoan groups use mitosis almost entirely. By mitosis and cell associations arise the elaborately diversified cell structures and functions found in such groups.

A diagramatic sketch of the representatives on a nuclear spirem in early synapsis stages of mitotic division in Spirogyra jugalis might show something like figure I I.

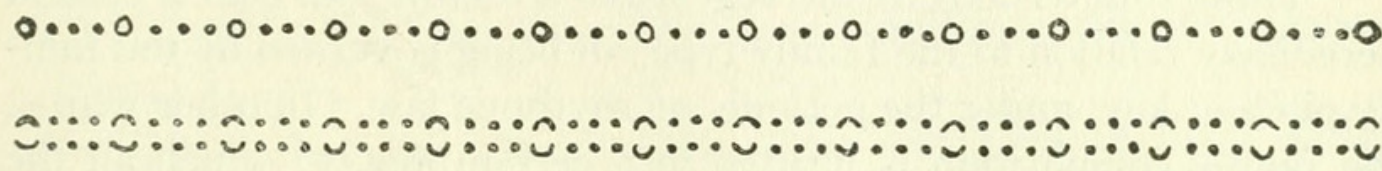

Fig. K.-Diagram illustrating binary division. The large dots of the upper row represent the chromosomes, the smaller dots the minor units on the skein. The lower row shows the division of all the components of the skein.

This has led up by easy stages to an understanding of the process of conjugation.

In conjugation the nuclei of both male and female sex cells prepare for remating by reducing the number of chromosomes, by which each loses its opposite sex elements; the new nucleus is composed of representatives from both parent chromosome groups, the character of the new cell person depending on the dominant factors represented at the union of the chromosome groups, modified constantly during its life by environment.

In parthenogenesis the ovum nucleus undergoes segmentation without conjugation and its attendant phenomena.

The body within the nucleus, called the nucleolus, plays an important part in the reformation of the vegetative structures of the cyto-plasm after mitosis. As it is composed of inbudded opposites of the vegetative groups, they would naturally be reproduced by its outbuddings.

The contents of this body are found in a variety of semi-fluid states, and is generally recognized by its peculiar staining properties, the contained substance being known as chromatin. 
In early vegetative stages of cell life, the nucleolus undergoes a remarkable series of binary divisions, producing within the nucleus a number of daughter bodies known as nucleosomes. These bodies enlarge by vacuolation, forming globular bodies, which migrate in many cases to the nuclear membrane, and in many other cases come to lie entirely outside of the membranes, to which they remain attached by filaments.

A further duplication of this cytosome group now occurs in many cases, the product of the first nucleolus reduction functioning as nuclei for a second generation, which in turn function as nuclei for a third generation, and these in turn produce other generations.

These subuivisions of the vegetative structures all bear a certain hereditary relation to the family type, all being governed by the family pitch or key, under the periodic or rhythmic law. In other words, the family composition is written in a certain key, to which all the factors bear a certain definite family relation, regardless of what the pitch of the keynote may be.

In all these generations of nucleosomes, there are constantly met certain heterogeneous divisions of the various elements, which would at first thought appear to upset the validity of the conclusion. The student of these matters will find plenty of examples for himself.

Mature thought brings us to believe that the keynotes are determined by the averages of the phenomena, not by individual departures from the family type. The averages form the dominant factors, while the variants form the secondary or weaker factors of the assemblage. This idea of averages gives us a good understanding of the results obtained by crossing species with a varying number of chromosomes.

According to Rosenberg, in the Botanical Gazette of September, I909, page 234, he has obtained most interesting results in this line with Drosera plants.

"In D. longifolia there are 40 chromosomes in the nuclei of the sporophyte, and 20 in the gametophyte, while in D. rotundifolia the numbers are 20 and Io respectively."

"In the hybrid called D. abovata the sporophyte shows regularly 30 chromosomes, the anticipated number." 
Rosenberg "crossed D. abovata with D. longifolia, and while usually there were no results (he) obtained a few embryos. These contained at least 33 chromosomes, and in one case 37 were counted. The theoretical number would be 35 ."

In the Botanical Gazette, Vol. 48, page 179, Gates gives the results of the cross between Oenothera lata, I4 chromosomes, and $O$. gigas, with 28 chromosomes; the resultant hybrids show 20 and 22. The theoretical number would be $2 \mathrm{I}$.

In figure 47 of the food storage cells of a Camponotus pupa, the nuclei will be seen full of nucleosomes derived from reduction divisions of the nucleolus. These nucleosomes are rapidly growing and becoming drawn outward into the cyto-plasm fluids, in which plasm are found the food products which they reorganize and store in metaphase.

Later in many cases, after these cyto-plastids have matured their special cytosome products in meta-phase, the contents of the plastids undergo anatosis reduction divisions.

The importance of close observation of the nucleosome bodies during the formation of vegetative bodies cannot be overestimated; probably all had their origin phylogenetically from this source, no matter how obscure their ontogenetic derivation may now be.

During mitosis or indirect cell division, the vegetative opposites forming the nucleolus are supposed to undergo binary division, and take their place as nucleosomes among the minor vegetative representatives on the nuclear reticulum; eventually they form the chromosomes.

The nucleus and nucleolus are reformed in ana-phase of mitosis by a reversal of the pro-stage.

The centrosome is a body which plays an important role in mitotic cell divisions. As the direct or binary divisions of the cell are carried on by the nucleolus, so the indirect division is initated by binary division of the centrosome body. The nucleolus disappears during mitotic cell division, and reappears at telophase; meanwhile the centrosomes are functionally active. Their functions are homologous, each functioning in its own peculiar style of cell division. 


\section{DESCRIPTION OF PLATES}

\section{Plate VI}

Fig. I. Cross section of the back thoracic muscles of a Tussock Moth pupa, 4 day stage of a female.

Fig. 2. Cross section of the same group two days later; all but two of the cells are now degenerating.

Fig. 3. Multinucleated muscle-cells from a Spider's leg.

Fig. 4. Cross section of the above muscle-cells from a Spider.

Fig. 5. Long section of the muscle-cells in a newly hatched Frog tadpole tail. The fibrils are undergoing longitudinal binary divisions.

Fig. 6. Long section of the back thoracic muscles of a Fly pupa, Sarchophaga, eight day stage.

\section{Plate VII}

Fig. 7. Muscle cells from a Salamander pup, showing the enormous numbers of cytosome fibrils which arise.

Fig. 8. Cross section of the above muscle-cells, showing the rapid increase of fibrils by division of cytosomes.

Fig. 9. Nucleosomes during early stages of sperm formation in Salamander testis.

Fig. Io. Formation of tails by growth of cytosomes in the tails of Salamander sperms.

Fig. II. The growth of cytosome fibrils into cilia. From the intestinal cells of an Ant pupa.

Fig. I2. The development of cytosome fibrils in the tails of Earthworm sperms. 


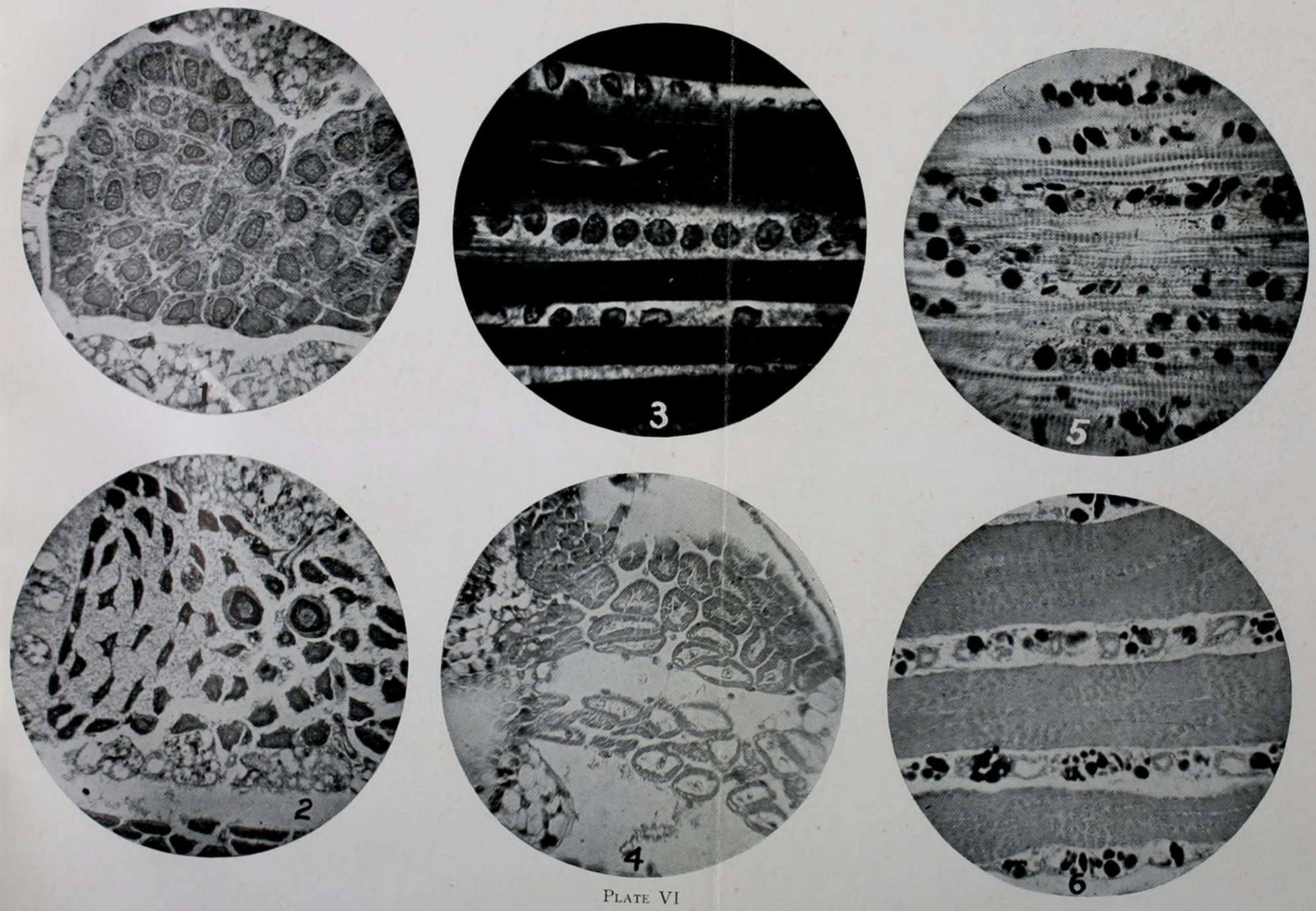



\section{$2 \mathrm{BHL}$ Biodiversity Heritage Library}

Roberts, E W. 1912. "The Modern Theory of the Cell as a Complex of Organized Units." Transactions 31, 85-113.

View This Item Online: https://www.biodiversitylibrary.org/item/86973

Permalink: https://www.biodiversitylibrary.org/partpdf/90699

\section{Holding Institution}

University of Toronto - Gerstein Science Information Centre

\section{Sponsored by}

University of Toronto

\section{Copyright \& Reuse}

Copyright Status: Not provided. Contact Holding Institution to verify copyright status.

This document was created from content at the Biodiversity Heritage Library, the world's largest open access digital library for biodiversity literature and archives. Visit BHL at https://www.biodiversitylibrary.org. 